\title{
Slater's Nonlocal Exchange Potential and Beyond
}

\author{
I. A. HOWARD, ${ }^{1}$ N. H. MARCH ${ }^{1,2}$ \\ ${ }^{1}$ Department of Physics, University of Antwerp, Groenenborgerlaan 171, B-2020 Antwerp, Belgium \\ ${ }^{2}$ Oxford University, Oxford, England
}

Received 30 July 2004; accepted 6 August 2004

Published online 4 November 2004 in Wiley InterScience (www.interscience.wiley.com).

DOI 10.1002/qua.20352

\begin{abstract}
The local density approximation (LDA) to the exchange potential $V_{x}(\mathbf{r})$, namely the $\rho^{1 / 3}$ electron gas form, was already transcended in Slater's 1951 paper.

Here, using Dirac's 1930 form for the exchange energy density $\epsilon_{x}(\mathbf{r})$, the Slater (Sl)

nonlocal exchange potential $V_{x}^{S I}(\mathbf{r})$ is defined by $2 \epsilon_{x}(\mathbf{r}) / \rho(\mathbf{r})$. In spherical atomic ions, say the Be or Ne-like series, this form $V_{x}^{S l}(\mathbf{r})$ already has the correct behavior in both $r \rightarrow 0$ and $r \rightarrow \infty$ limits when known properties of the exchange energy density $\epsilon_{x}(\mathbf{r})$ and the ground-state electron density $\rho(\mathbf{r})$ are invoked. As examples, some emphasis will first be given to the use of the so-called $1 / Z$ expansion in such spherical atomic ions, for which analytic results can be obtained for both $\epsilon_{x}(\mathbf{r})$ and $\rho(\mathbf{r})$ as the atomic number $Z$ becomes large. The usefulness of the $1 / Z$ expansion is directly demonstrated for the $U$ atomic ion with 18 electrons by comparison with the optimized effective potential prediction. A rather general integral equation for the exchange potential is then proposed. Finally, without appeal to large $Z$, two-level systems are considered, with specific reference to the Be atom and to the $\mathrm{LiH}$ molecule. In all cases treated, the Slater potential $V_{x}^{S l}(\mathbf{r})$ is a valuable starting point, even though it needs appreciable quantitative corrections reflecting directly atomic shell structure. (c) 2004 Wiley Periodicals, Inc. Int J Quantum
\end{abstract} Chem 102: 64-71, 2005

Key words: exchange potential; density matrix; optimized effective potential

Correspondence to: I. A. Howard; e-mail: howard@ruca.ua.ac.be This manuscript was submitted for the 2003 Herbert Jones issue, but due to an error at the editor's office (not by the author), this manuscript is being published in this issue.

This article is dedicated to the memory of Professor Herbert Jones.

Contract grant sponsors: Instituut voor de Aanmoediging van Innovatie door Wetenschap en Technologie (IWT-Flemish region); Office of Naval Research (ONR). 


\section{Background and Outline}

C urrent methods of calculation in density functional theory (DFT) solve one-electron Schrödinger equations (the so-called Slater-Kohn-Sham equations [1, 2]) into which an effective potential energy $V(\mathbf{r})$ is introduced. This potential, since it is designed to obtain the exact ground-state electron density $\rho(\mathbf{r})$ as

$$
\rho(\mathbf{r})=2 \sum_{\text {occupied states }} \phi_{i}^{*}(\mathbf{r}) \phi_{i}(\mathbf{r}),
$$

must contain a contribution from quantum mechanical exchange $(x)$ and correlation (c) interactions. Then $V(\mathbf{r})$ can be written

$$
V(\mathbf{r})=V_{n}(\mathbf{r})+V_{e s}(\mathbf{r})+V_{x c}(\mathbf{r}),
$$

where $V_{n}(\mathbf{r})$ is the potential from the nuclei in the atom, molecule or cluster under consideration, $V_{e s}(\mathbf{r})$ is the electrostatic potential created by the electron distribution $\rho(\mathbf{r})$, while $V_{x c}(\mathbf{r})$ subsumes the exchange and correlation contributions. It is known (see, e.g., Levy and March [3]) that $V_{x c}(\mathbf{r})$ can be rigorously separated into two parts: the exchange potential $V_{x}(\mathbf{r})$ and the correlation contribution $V_{c}(\mathbf{r})$. It is now generally accepted that in many systems of current interest for quantum chemical studies, the exchange effects are an order of magnitude larger than those from correlation. Thus, to obtain good approximations to the orthonormal orbitals $\phi_{i}(\mathbf{r})$ in Eq. (1.1), written for spin-compensated electronic assemblies to which we confine ourselves throughout the present study, it is essential to calculate the exchange potential $V_{x}(\mathbf{r})$ in a fully quantitative form, and to make the (presently unavoidable) approximations in the "small" correlation contribution $V_{c}(\mathbf{r})$ to the potential $V(\mathbf{r})$ in Eq. (1.2).

\subsection{DIRAC DENSITY MATRIX AND EXCHANGE ENERGY DENSITY}

In the present investigation, it is important to make an off-diagonal generalization of Eq. (1.1), namely to define the Dirac [4] density matrix $\gamma\left(\mathbf{r}, \mathbf{r}^{\prime}\right)$ by

$$
\gamma\left(\mathbf{r}, \mathbf{r}^{\prime}\right)=2 \sum_{\text {occupied states }} \phi_{i}^{*}(\mathbf{r}) \phi_{i}\left(\mathbf{r}^{\prime}\right) .
$$

Then, as already known to Dirac [4], the total exchange energy, denoted by $E_{x}$ below, is given explicitly by

$$
E_{x}=-\frac{e^{2}}{4} \int \frac{\gamma^{2}\left(\mathbf{r}, \mathbf{r}^{\prime}\right)}{\left|\mathbf{r}-\mathbf{r}^{\prime}\right|} d \mathbf{r} d \mathbf{r}^{\prime}
$$

In principle (see, e.g., Ref. [2]), the exchange potential $V_{x}(\mathbf{r})$ is given by the functional derivative of $E_{x}$ with respect to the ground-state density $\rho(\mathbf{r})$. But this route is not yet practicable in quantum chemical problems, as it requires explicitly the Dirac density matrix $\gamma\left(\mathbf{r}, \mathbf{r}^{\prime}\right)$ as a functional of its diagonal element $\rho(\mathbf{r})$. Presently, such knowledge is only available for a small number of analytically solvable models $[5,6]$.

Therefore, in recent work, the present authors have focused on calculating the exchange energy density $\epsilon_{x}(\mathbf{r})$ [5] using the leading term in the socalled $1 / Z$ expansion in atomic theory $[7,8]$, where $Z$ is the atomic number. Specifically, for atomic ions with closed $K+L$ shells and large $Z$, the nonrelativistic Dirac density matrix needed to calculate $\epsilon_{x}(\mathbf{r})$, defined in a natural (but not unique) way from the Dirac result (1.4) as

$$
\epsilon_{x}(\mathbf{r})=-\frac{e^{2}}{4} \int \frac{\gamma^{2}\left(\mathbf{r}, \mathbf{r}^{\prime}\right)}{\left|\mathbf{r}-\mathbf{r}^{\prime}\right|} d \mathbf{r}^{\prime},
$$

was constructed from hydrogenic wave functions (leading term in $1 / Z$ expansion). The asymptotic form at large $r$ obtained from the analytic form of $\epsilon_{x}(\mathbf{r})$ given in Ref. [5] was shown numerically to approach quickly the asymptotic form given earlier by one of us [9]:

$$
\boldsymbol{\epsilon}_{x}(r)=-\frac{1}{2} \frac{e^{2}}{r} \rho(r): \quad r \rightarrow \infty .
$$

This asymptotic form (1.6) already demonstrates the limitations of the local density approximation (LDA), i.e., electron gas results. For example, as Dirac [4] first proved (see also Ref. [10]),

$$
\epsilon_{x}^{\mathrm{LDA}}(\mathbf{r})=-c_{x} \rho^{4 / 3}(\mathbf{r}): \quad c_{x}=\frac{3}{4} e^{2}\left(\frac{3}{\pi}\right)^{1 / 3} .
$$

Comparison of Eqs. (1.6) and (1.7) far from the nucleus of a spherically symmetrical atomic ion (e.g., the Ne-like series) shows that while the LDA result (1.7) contains only one characteristic 
"length," namely $\rho(r)^{-1 / 3}$, the correct Eq. (1.6) contains two: $\rho(r)^{-1 / 3}$ as with LDA, but also the electron-nucleus separation $r$. Put another way, $\epsilon_{x}(r) /$ $\rho(r)$ falls off in this example as $-(1 / 2) e^{2} / r$ at large $r$, this slow falloff to be contrasted with the LDA rapid exponential decay as $\rho(r)^{1 / 3}$.

\subsection{SLATER'S NONLOCAL EXCHANGE POTENTIAL}

Slater [1] had already anticipated this difficulty (see also the later study of Kleinman [11, 12]) and proposed to define a nonlocal exchange potential, denoted by $V_{x}^{S l}(\mathbf{r})$ below, by writing, in conformity with the classical electrostatic energy $U_{e s}$ given by

$$
\begin{gathered}
U_{e s}=\frac{e^{2}}{2} \int \frac{\rho(\mathbf{r}) \rho\left(\mathbf{r}^{\prime}\right)}{\left|\mathbf{r}-\mathbf{r}^{\prime}\right|} d \mathbf{r} d \mathbf{r}^{\prime}=\frac{e^{2}}{2} \int \rho(\mathbf{r}) V_{e s}(\mathbf{r}) d \mathbf{r}, \\
E_{x}=\frac{e^{2}}{2} \int \rho(\mathbf{r}) V_{x}^{S l}(\mathbf{r}) d \mathbf{r} .
\end{gathered}
$$

Comparing Eq. (1.9) with the Dirac result (1.4) leads, naturally enough, to Slater's nonlocal exchange potential as

$$
V_{x}^{S l}(\mathbf{r})=-\frac{1}{2 \rho(\mathbf{r})} \int \frac{\gamma^{2}\left(\mathbf{r}, \mathbf{r}^{\prime}\right)}{\left|\mathbf{r}-\mathbf{r}^{\prime}\right|} d \mathbf{r}^{\prime}
$$

In view of our own study of the exchange energy density $\epsilon_{x}(\mathbf{r})$ in Eq. (1.5), we find it convenient to rewrite Eq. (1.10) in the form

$$
V_{x}^{S l}(\mathbf{r})=\frac{2 \epsilon_{x}(\mathbf{r})}{\rho(\mathbf{r})},
$$

which we can adopt as an alternative "definition" of the Slater nonlocal exchange potential. The nonlocality referred to, in order to distinguish it from the $\rho^{1 / 3}$ LDA result obtained by differentiating Eq. (1.7) with respect to the density $\rho(\mathbf{r})$, is immediately apparent from the definition (1.10) due to Slater [1], but also follows, as it must, from the equivalent definition (1.11) when the nonlocal (Dirac) form (1.5) of $\epsilon_{x}(\mathbf{r})$ is invoked.

One favorable property of the Slater potential (1.11) follows from the asymptotic form (1.6) for spherical atomic ions, namely that $V_{x}(r) \rightarrow-e^{2} / r$ at large $r$ in such electronic assemblies. This treats exactly the asymptotic property of correcting the self-interaction included in $V_{e s}(\mathbf{r})$ in Eq. (1.2), since the electrostatic interaction is calculated from $\rho(\mathbf{r})$, which includes all $N$ electrons in the system. Indeed, $V_{x c}(r) \rightarrow-e^{2} / r$ in such spherical atomic ions, so $V_{x}^{S l}(r)$ already takes care of that exact limiting property in these admittedly simple spherical electron density ions.

Following this survey, the outline of the remainder of this article is as follows. In Section 2, the Dirac density matrix entering Eq. (1.5) is approximated by the leading term in the $1 / Z$ expansion. The exchange energy density can then be calculated for atomic ions for 4,10, and 18 electrons, and these results are used to obtain the Slater exchange potential. This is then compared with the optimized effective potential, stemming from the early studies of Sharp and Horton [13] and of Talman and Shadwick [14]. Section 3 then focuses on a formally exact integral equation for $V_{x}(\mathbf{r})$, which, at least in principle, allows "corrections" to the Slater exchange potential $V_{x}^{S l}(\mathbf{r})$ in Eq. (1.11) to be exhibited. However, a function entering this integral equation [cf. Eqs. (3.1) and (3.2) below] has to be approximated. After referring to one, relatively simple, approximation worked out for Be (see Fig. 3), a more general proposal is made that establishes contact with the optimized effective potential [13, 14] already referred to. Returning to two-level systems such as Be in Section 4, a further potential proposed by Harbola and Sahni [15] is discussed in relation to the Slater exchange potential $V_{x}^{S l}(\mathbf{r})$. It should be noted at this point that the original Harbola-Sahni proposal [15] was criticized as involving a path integral which depended on the path chosen [16]. However, using low-order density matrices, Holas and March [17] derived an exact expression for the force $-\partial V_{x c}(\mathbf{r}) / \partial \mathbf{r}$ associated with the exchange-correlation potential $V_{x c}(\mathbf{r})$ introduced in Eq. (1.2). Using their study, but now for $V_{x}(\mathbf{r})$, Levy and March [3] exhibited "kinetic corrections" to the approximation $V_{x}^{H S}(\mathbf{r})$, which restored the path independence. Finally, Section 5 constitutes a summary as well as some suggestions for future work that may prove fruitful.

\section{Limiting Results for 4,10 , and 18 Electron Atomic Ions for Large Atomic Number $Z$ in Nonrelativistic Theory}

One can obtain analytically the total exchange energy $E_{x}$ in Eq. (1.4) when the Dirac density matrix 
is replaced by its large- $Z$ limit, built from hydrogen-like wave functions. We first summarize the results for electron number $N$ equal to 4 and 10 .

\subsection{LIMITING LARGE-Z RESULTS FOR CONFIGURATION $(1 s)^{2}(2 s)^{2}$}

For $N=4$, we have the limiting form

$$
E_{x}=-0.81929 Z \text {, }
$$

with the corresponding exchange energy density $\epsilon_{x}(r)$

$$
\begin{array}{r}
\boldsymbol{\epsilon}_{x}(r)=\frac{Z^{3}}{8 \pi} \exp (-Z r)\left[-\frac{1}{r}+Z-\frac{Z^{2} r}{4}\right] \\
+\frac{Z^{3}}{8 \pi} \exp (-2 Z r)\left[-\frac{7}{r}-\frac{Z}{4}-\frac{Z^{2} r}{4}+\frac{Z^{3} r^{2}}{32}-\frac{Z^{4} r^{3}}{32}\right. \\
\left.+\frac{Z^{5} r^{4}}{64}\right]+\frac{Z^{4}}{9 \pi} \exp (-3 Z r)\left[-\frac{4}{3}-\frac{4 Z r}{3}+Z^{2} r^{2}\right] \\
+\frac{Z^{3}}{\pi} \exp (-4 Z r)\left[\frac{1}{r}+Z\right] .
\end{array}
$$

The electron density for the $(1 s)^{2}(2 s)^{2}$ configuration is

$$
\begin{aligned}
\rho(r)=\frac{Z^{3}}{16 \pi} & \exp (-Z r) \\
\times & {\left[32 \exp (-Z r)+4-4 Z r+Z^{2} r^{2}\right] . }
\end{aligned}
$$

Hence, the Slater exchange potential $V_{x}^{S l}(r)=2 \epsilon_{x}(r) /$ $\rho(r)$ is known in the large- $Z$ limit. One simple analytical consequence of Eqs. (2.2) and (2.3) for $V_{x}^{S l}(r)$ is that the $r \rightarrow 0$ limit has the form

$$
\lim _{r \rightarrow 0} V_{x}^{S l}(r)=-\frac{1019}{972} Z,
$$

the corresponding limit for the exchange density being readily obtained from Eq. (2.2) as

$$
\lim _{r \rightarrow 0} \epsilon_{x}(r)=-\frac{1019}{864} \frac{Z^{4}}{\pi}
$$

On the basis of numerical results for 10 electrons and $Z=92$, we expect $V_{x}^{S l}(r)$ calculated from Eqs. (1.11), (2.2), and (2.3) to be a good approximation to

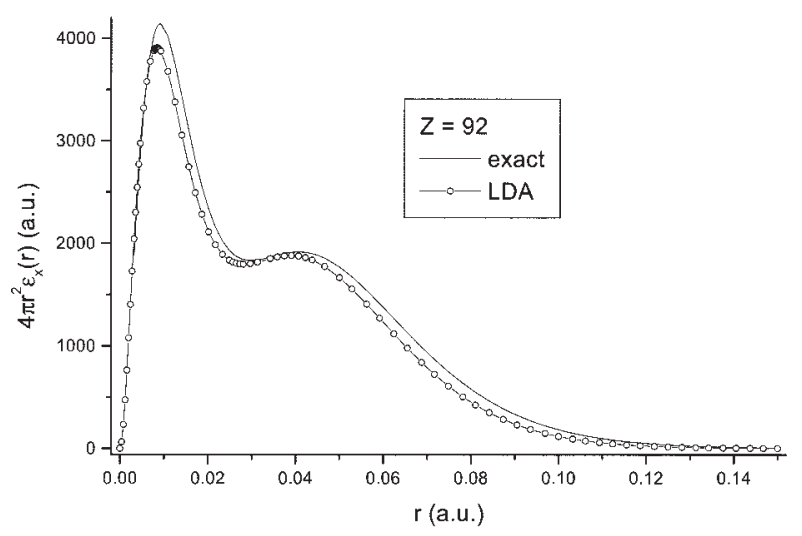

FIGURE 1. Plot of radial form $4 \pi r^{2} \epsilon_{x}(r)$ of exchange energy density defined by Eq. (1.5) for $Z=92$ and for 10 electrons in the hydrogenic (nonrelativistic) limit of large Z. For comparison, the LDA in Eq. (1.7) is shown. Although the point-point agreement seems quite good, the total exchange energy of LDA is some $8 \%$ in error. Redrawn from Ref. [5].

the true exchange potential for the uranium atom stripped of all but four of its electrons.

\subsection{LIMITING LARGE-Z FORMS FOR Ne- LIKE CONFIGURATION $(1 s)^{2}(2 s)^{2}(2 p)^{6}$}

Since, near the nucleus at $r=0, \rho(r)$ and $\epsilon_{x}(r)$ are determined by the same $s$-states, in the large- $Z$ limit the results (2.4) and (2.5) immediately apply to the configuration $(1 s)^{2}(2 s)^{2}(2 p)^{6}$. The analytical form of $\epsilon_{x}(r)$ for this 10-electron case is recorded in Ref. [5]. The radial form $4 \pi r^{2} \epsilon_{x}(r)$ is plotted in Figure 1, where it is compared with the corresponding quantity from the LDA form (1.7), using exactly the same density $\rho(r)$. In spite of the remarkable point-topoint agreement, the total exchange energy $E_{x}$ using LDA is in error by $\sim 8 \%$ and this is an unacceptably large energy for chemical applications. The correct limiting large- $Z$ form of $E_{x}$ for configuration $(1 s)^{2}(2 s)^{2}(2 p)^{6}$ is given by [5]

$$
E_{x}=-1.74788 Z,
$$

in atomic units $\left(e^{2} / a_{0}=1 ; a_{0}=\hbar^{2} / \mathrm{me}^{2}\right)$. It is also straightforward to verify the cusp-like condition [18]:

$$
\left.\frac{\partial \epsilon_{x}(r)}{\partial r}\right|_{r=0}=-\left.\frac{2 Z}{a_{0}} \epsilon_{x}(r)\right|_{r=0}: \quad a_{0}=\hbar^{2} / m e^{2}
$$




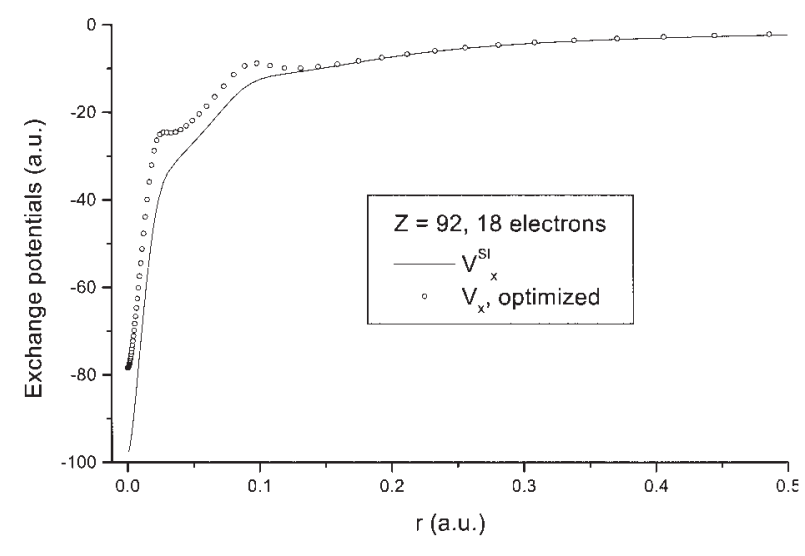

FIGURE 2. Compares hydrogenic limit for $Z=92$ and 18 electrons of the Slater exchange potential with the optimized effective potential. Redrawn from Ref. [19].

and that for the Slater potential

$$
\lim _{r \rightarrow 0} \frac{\partial V_{x}^{S l}(r)}{\partial r}=0 .
$$

\subsection{SIMILAR LIMITING FORMS FOR Ar-LIKE CONFIGURATION}

Very recently, Howard et al. [19] have considered 18-electron Ar-like atomic ions. For this case, we have in the large- $Z$ limit

$$
E_{x}=-2.33387 Z \text {. }
$$

Forms (2.7) and (2.8) are again satisfied. For the specific case of uranium, with $Z=92$, the atomic ion with 18 electrons has a Slater exchange potential $V_{x}^{S l}(r)$ shown in Figure 2. This is compared with the optimized effective potential of Refs. [13] and [14] in this figure. In the region near $r=0$, where the deviations from the Slater exchange potential are largest, the nuclear potential $V_{n}(r)=-Z^{2} / r$ with $Z=92$ in the present example of course dominates $V(r)$ defined in Eq. (1.2). However, the atomic shell structure is clearly reflected in the corrections shown to the Slater exchange potential in Figure 2.

\section{Exact Integral Equation for Exchange-Only Potential $V_{x}(R)$}

In recent work, Howard and March [20] have given a formally exact generalization of the integral equation of Della Sala and Görling [21] for the exact exchange-only potential $v_{x}(\mathbf{r})$ of DFT. This generalized equation [see Eq. (3.1) below] relates $V_{x}(\mathbf{r})$ to the Slater exchange potential $V_{x}^{S l}(\mathbf{r})$, which is directly related to the Dirac exchange energy density [4] $\epsilon_{x}(\mathbf{r})$ defined by Eq. (1.5) above. Here $\gamma / 2$ is an idempotent singleparticle density matrix with half the ground-state electron density $\rho(\mathbf{r})$ as its diagonal element. The Slater potential is then given by Eq. (1.11). The integral equation of Howard and March [20] then reads

$$
\begin{aligned}
& V_{x}(\mathbf{r})-V_{x}^{S l}(\mathbf{r})=\frac{1}{4 \rho(\mathbf{r})} \int \gamma^{2}\left(\mathbf{r}, \mathbf{r}^{\prime}\right) V_{x}\left(\mathbf{r}^{\prime}\right) d \mathbf{r}^{\prime} \\
& -\frac{1}{8 \rho(\mathbf{r})} \int \frac{\gamma\left(\mathbf{r}, \mathbf{r}^{\prime}\right) \gamma\left(\mathbf{r}^{\prime}, \mathbf{r}^{\prime \prime}\right) \gamma\left(\mathbf{r}^{\prime \prime}, \mathbf{r}\right)}{\left|\mathbf{r}^{\prime}-\mathbf{r}^{\prime \prime}\right|} d \mathbf{r}^{\prime} d \mathbf{r}^{\prime \prime}+\frac{P(\mathbf{r})}{\rho(\mathbf{r})},
\end{aligned}
$$

where

$$
\int P(\mathbf{r}) d \mathbf{r}=0 .
$$

In Ref. [20], an ansatz was made for $P(\mathbf{r})$ for the Be-like atomic ion sequence. Exploiting the orthogonality of the $1 s$ wave function, $\psi_{1}(r)$, and the $2 s$ function $\psi_{2}(r)$ led to the assumption that $P(\mathbf{r})$ was proportional to the product $\psi_{1}(r) \psi_{2}(r)$, which evidently satisfies the integral condition (3.2). This led to the results for the exchange potential of $\mathrm{Be}$ shown in Figure 3.

Here we propose a much more general ansatz for $P(\mathbf{r})$, which appeals directly to the integral equation given by Talman [22] for the optimized effective exchange potential (OEP), which has recently been used by Howard et al. [19] to calculate approximate exchange potentials for the 10-electron Ne-like series of atomic ions as well as Figure 2 for 18 electrons. This integral equation for $v_{x}(\mathbf{r})$ reads (see Eqs. (10) and (11) of Ref. [22]):

$$
\int K\left(\mathbf{r}, \mathbf{r}^{\prime}\right) V_{x}\left(\mathbf{r}^{\prime}\right) d \mathbf{r}^{\prime}=Q(\mathbf{r}),
$$

where both $K\left(\mathbf{r}, \mathbf{r}^{\prime}\right)$ and $Q(\mathbf{r})$ involve a single-particle Green function. Using again orthogonality properties, discussed already by Talman [22], it is straightforward to show that $\int Q(\mathbf{r}) d \mathbf{r}=0$. It is this result that prompts the new ansatz we propose here, namely that $P(\mathbf{r})$ in the formally exact integral Eq. (3) of Howard and March [20] is a constant, $\lambda$ 


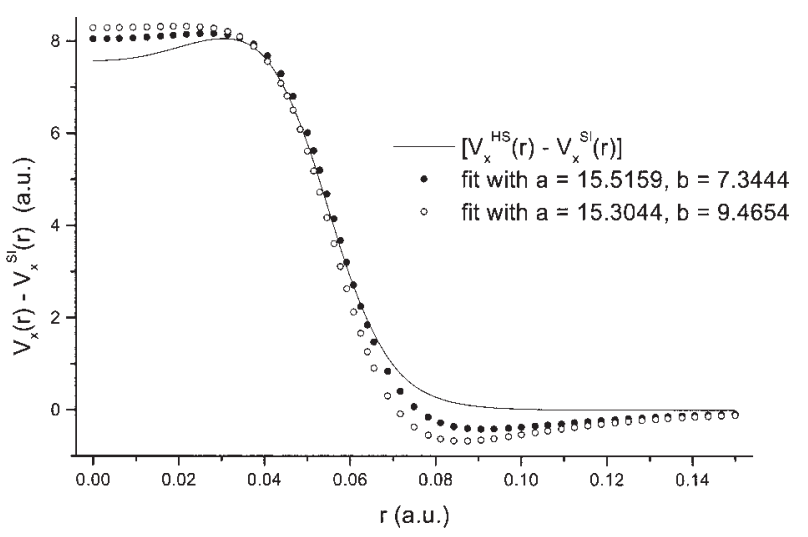

FIGURE 3. Illustrates results for $V_{x}(r)-V_{x}^{S /}(r)$ for the Be atom using the integral equation (3.1) with the choice of $P(r)=$ const. $\times \psi_{1}(r) \psi_{2}(r)$, with $1 \equiv 1 s$ and $2 \equiv 2 s$, for the configuration $(1 s)^{2}(2 s)^{2}$. The difference between the Harbola-Sahni exchange potential $V_{x}^{H S}(r)$ and the nonlocal Slater potential $V_{x}^{S I}(r)$ is shown for comparison (see also Section 4). The two choices of "weighting parameters" $a$ and $b$ in Ref. [20] are designed to satisfy the virial relation of Levy and Perdew [23]. Redrawn from Ref. [20].

say, times $Q(\mathbf{r})$. One then finds almost immediately the "hybrid" integral equation

$$
\begin{aligned}
V_{x}(\mathbf{r})- & V_{x}^{S l}(\mathbf{r}) \\
= & \frac{1}{4 \rho(\mathbf{r})} \int\left[\gamma^{2}\left(\mathbf{r}, \mathbf{r}^{\prime}\right)-4 \pi \lambda K\left(\mathbf{r}, \mathbf{r}^{\prime}\right)\right] V_{x}\left(\mathbf{r}^{\prime}\right) d \mathbf{r}^{\prime} \\
& -\frac{1}{8 \rho(\mathbf{r})} \int \frac{\gamma\left(\mathbf{r}, \mathbf{r}^{\prime}\right) \gamma\left(\mathbf{r}^{\prime}, \mathbf{r}^{\prime \prime}\right) \gamma\left(\mathbf{r}^{\prime \prime}, \mathbf{r}\right)}{\left|\mathbf{r}^{\prime}-\mathbf{r}^{\prime \prime}\right|} d \mathbf{r}^{\prime} d \mathbf{r}^{\prime \prime}
\end{aligned}
$$

The final step needed is to determine the $\mathbf{r}$-independent quantity $\lambda$, measuring the "hybrid" character of Eq. (6). This we now fix by insisting that the Levy-Perdew virial-like relation [23]

$$
E_{x}=-\int \mathbf{r} \cdot \frac{\partial V_{x}(\mathbf{r})}{\partial \mathbf{r}} \rho(\mathbf{r}) d \mathbf{r}
$$

is exactly satisfied, where $E_{x}$ is the total exchange energy obtained from Eq. (1.4) as

$$
E_{x}=\int \boldsymbol{\epsilon}_{x}(\mathbf{r}) d \mathbf{r}
$$

As the simplest example of the use of Eq. (2.14), let us use it for spherically symmetric atomic ions, such as the Be or Ne-like series already referred to. Then, integrating by parts, one finds from Eq. (3.5) that

$$
E_{x}=3 \int V_{x}(r) \rho(r) d \mathbf{r}+4 \pi \int_{0}^{\infty} r^{3} V_{x}(r) \frac{d \rho(r)}{d r} d r
$$

Inserting the ansatz (3.4) into Eq. (3.7), one obtains a further equation from which $\lambda$ can be determined iteratively as the solution of the integral Eq. (3.4) proceeds.

\section{Relation Between Harbola-Sahni and Slater Exchange Potentials for the Be Atom, Based on the Hartree-Fock Ground-State Density for Two-Level Systems}

So far we have dealt with exchange-only DFT. However, since for atoms and molecules the Hartree-Fock (HF) density is normally of high quality, as known from comparison with x-ray and electron diffraction measurements, we shall summarize in this penultimate section the way the difference between the Slater exchange potential, based now on the HF electron density, and the Harbola-Sahni potential discussed above, can be estimated for the two-level systems such as the Be atom or the $\mathrm{LiH}$ molecule.

The central result used below (see also Ref. [24]) is that

$$
\frac{\partial}{\partial r}\left[V_{x}^{\mathrm{HS}}(\mathbf{r})-V_{x}^{S l}(\mathbf{r})\right]=-\theta^{\prime}(\mathbf{r}) F(\mathbf{r})
$$

where $F(\mathbf{r})$ denotes the "Fock operator" term, while $\theta(\mathbf{r})$ is the phase defined in terms of the HF wave functions $\psi_{1}(\mathbf{r})$ and $\psi_{2}(\mathbf{r})$ for the two-level system under investigation by [25]:

$$
\psi_{1}(\mathbf{r})=\rho^{1 / 2}(\mathbf{r}) \cos \theta(\mathbf{r})
$$

and

$$
\psi_{2}(\mathbf{r})=\rho^{1 / 2}(\mathbf{r}) \sin \theta(\mathbf{r}),
$$

with $\rho(\mathbf{r})$ now the HF density as discussed above. Hence, from the HF wave functions $\psi_{1}(\mathbf{r})$ and $\psi_{2}(\mathbf{r})$, we have

$$
\tan \theta(\mathbf{r})=\psi_{2}(\mathbf{r}) / \psi_{1}(\mathbf{r})
$$

and hence $\theta^{\prime}(\mathbf{r})$ needed in Eq. (4.1) can be obtained. 
Turning to the "Fock operator" term $F(\mathbf{r})$, this is determined by $\rho(\mathbf{r})$ and the phase $\theta(\mathbf{r})$ by [24]:

$$
F(\mathbf{r})=1 / 2 \int d \mathbf{r}^{\prime} \frac{\rho\left(\mathbf{r}^{\prime}\right) \sin \left[2 \theta(\mathbf{r})-2 \theta\left(\mathbf{r}^{\prime}\right)\right]}{\left|\mathbf{r}-\mathbf{r}^{\prime}\right|}
$$

We have not carried out numerical evaluation of $V_{x}^{H S}(\mathbf{r})-V_{x}^{S l}(\mathbf{r})$ for the Be atom, since we anticipate that the result derivable from Eqs. (4.1), (4.4), and (4.5) will be very close to that recorded in Figure 3.

To close this section, we note that the above procedure could, in principle, be applied to the "two-level" molecule LiH. However, the "kinetic" corrections of Ref. [3] would then have to be added to the Harbola-Sahni exchange potential.

\section{Summary and Proposed Future Directions}

We have stressed here both the considerable merits, and some quantitative limitations, of the Slater exchange potential $V_{x}^{S l}(\mathbf{r})$ defined in Eq. (1.11). Thus, in Figure 2, analytic knowledge of the density $\rho(r)$ and the exchange density $\epsilon_{x}(r)$ appearing in this equation in the large- $Z$ limit is used to compare $V_{x}^{S l}(r)$ with the optimized effective potential prediction for $Z=92$ and 18 electrons. The general features of $V_{x}^{S l}(r)$ are seen to be correct, although some modifications are called for to make the Slater potential fully quantitative.

In Section 3, we have made a proposal for an integral equation for the exchange-only potential $V_{x}(\mathbf{r})$. Equation (3.1) is a formally exact equation, but the function $P(\mathbf{r})$ on the right-hand side is not yet fully specified, although any correct choice must satisfy the "sum rule" given in Eq. (3.2). For $\mathrm{Be}$, the choice that $P(\mathbf{r})$ is proportional to the product of the $1 s$ and $2 s$ wave functions satisfies Eq. (3.2) because of wave function orthogonality, and consequences of this assumption for $P(\mathbf{r})$ are shown in Figure 3. We note here that Eq. (3.1) is a formally exact generalization of the study of Della Sala and Görling [21]; their work showing that $P(\mathbf{r})=0$ if one makes the (oversimplistic) assumption that the Hartree-Fock and Slater-Kohn-Sham determinants are equal.

A more general proposal is then made for $P(\mathbf{r})$, which directly invokes the integral Eq. (3.3) of the optimized effective potential method, as written, for example, by Talman [22], but now involving a free parameter $\lambda$. We believe it will be interesting in the future to study the results obtained for the correction $V_{x}(\mathbf{r})-V_{x}^{S l}(\mathbf{r})$ to the Slater exchange potential, when $\lambda$ is chosen to satisfy the Levy-Perdew virial-like relation (3.5).

\section{Appendix: Ground-State Density $\rho^{S I}(r)$ Generated for Spherical Atomic Ions by the Slater Nonlocal Exchange Potential}

We are here interested in studying $\rho^{S l}(r)$, the spherical atomic ion ground-state electron density, generated by the total potential $V^{S l}(r)$ given by

$$
V^{S l}(r)=-\frac{Z e^{2}}{r}+V_{e s}^{S l}(r)+V_{x}^{S l}(r) .
$$

Thus, applying the Laplacian operator to $V^{S l}(r)$ we find, excluding the nucleus at $r=0$,

$$
\nabla^{2} V^{S l}(r)=\nabla^{2} V_{e s}^{S l}(r)+\nabla^{2} V_{x}^{S l}(r) .
$$

But Poisson's equation yields

$$
\nabla^{2} V^{S l}(r)=4 \pi \rho^{S l}(r)+\nabla^{2} V_{x}^{S l}(r) .
$$

Next we insert Eq. (1.11) into the right-hand side of Eq. (A.3) to find

$$
\nabla^{2} V^{S l}(r)=4 \pi \rho^{S l}(r)+2 \frac{d^{2}}{d r^{2}}\left[\frac{\epsilon_{x}}{\rho}\right]+\frac{4}{r} \frac{d}{d r}\left[\frac{\epsilon_{x}}{\rho}\right] .
$$

Next we have

$$
\frac{d}{d r}\left[\frac{\epsilon_{x}}{\rho}\right]=-\frac{\rho^{\prime}}{\rho^{2}} \epsilon_{x}+\frac{\epsilon_{x}^{\prime}}{\rho}
$$

and therefore

$$
\frac{d^{2}}{d r^{2}}\left[\frac{\epsilon_{x}}{\rho}\right]=\frac{2 \rho^{\prime 2}}{\rho^{3}} \epsilon_{x}-\frac{2 \rho^{\prime}}{\rho^{2}} \epsilon_{x}^{\prime}+\frac{\epsilon_{x}^{\prime \prime}}{\rho}-\epsilon_{x} \frac{\rho^{\prime \prime}}{\rho^{2}} .
$$

Hence, using Eqs. (A.5) and (A.6) in Eq. (A.4), we find 


$$
\begin{aligned}
4 \pi \rho^{S l}(r)=\nabla^{2} V^{S l}(r) & -\frac{4 \rho^{\prime 2}}{\rho^{3}} \epsilon_{x}+\frac{4 \rho^{\prime}}{\rho^{2}} \epsilon_{x}^{\prime}-\frac{2 \epsilon_{x}^{\prime \prime}}{\rho} \\
& +\frac{2 \epsilon_{x} \rho^{\prime \prime}}{\rho^{2}}+\frac{4 \rho^{\prime}}{r \rho^{2}} \epsilon_{x}-\frac{4 \epsilon_{x}^{\prime}}{r \rho} .
\end{aligned}
$$

To proceed further, we invoke the concept of the Pauli potential $V_{P}(r)$ to write

$\nabla^{2}\left[\rho^{S l}(r)\right]^{1 / 2}+2\left[\epsilon-V^{S l}(r)-V_{P}^{S l}(r)\right]\left[\rho^{S l}(r)\right]^{1 / 2}=0$.

Dividing Eq. (A.8) throughout by $\left[\rho^{S l}(r)\right]^{1 / 2}$, we can use the result to eliminate $V^{S l}(r)$ from Eq. (A.7) to obtain

$$
\begin{aligned}
4 \pi \rho^{S l}(r)-\frac{1}{2} \frac{\nabla^{2}\left[\rho^{S l}(r)\right]^{1 / 2}}{\left[\rho^{S l}(r)\right]^{1 / 2}}+\nabla^{2} V_{P}^{S l}(r) & \\
=-4 \frac{\left[\rho^{S l}(r)^{\prime}\right]^{2} \epsilon_{x}(r)}{\left[\rho^{S l}(r)\right]^{3}}+ & \frac{4 \rho^{S l}(r)^{\prime} \epsilon_{x}^{\prime}(r)}{\left[\rho^{S l}(r)\right]^{2}}-\frac{2 \epsilon_{x}^{\prime \prime}(r)}{\rho^{S l}(r)}+\frac{2 \epsilon_{x} \rho^{S l}(r)^{\prime \prime}}{\left[\rho^{S l}(r)\right]^{2}} \\
& +\frac{4 \rho^{S l}(r)^{\prime} \epsilon_{x}(r)}{r\left[\rho^{S l}(r)\right]^{2}}-\frac{4 \epsilon_{x}^{\prime}(r)}{r \rho^{S l}(r)} . \quad \text { (A.9) }
\end{aligned}
$$

The $V_{P}^{S l}(r)$ definition can be given as

$$
V_{P}^{S l}(r)=\left.\frac{\delta T_{s}}{\delta \rho(r)}\right|_{\rho=\rho^{S l}}-\left.\frac{\delta T_{W}}{\delta \rho(r)}\right|_{\rho=\rho^{S l}} .
$$

This affords an explicit, although approximate, example of the intimate relationship between singleparticle kinetic energy density and exchange energy density, discussed earlier by March and Santamaria [26] and by Parr et al. [27].

\section{ACKNOWLEDGMENTS}

The authors thank Professors A. Holas and J. D. Talman for invaluable help. I. A. H. acknowledges support from the IWT-Flemish region under grant no. IWT-161. N. H. M. acknowledges partial financial support from ONR for work on density functional theory. Special thanks are due to Dr. P.
Schmidt of that Office for motivation and his continuing interest.

\section{References}

1. Slater, J. C. Phys Rev 1951, 81, 385.

2. Kohn, W.; Sham, L. J. Phys Rev A: At Mol Opt Phys 1965, $140,1133$.

3. Levy, M.; March, N. H. Phys Rev A: At Mol Opt Phys 1997, 55, 1885.

4. Dirac, P. A. M. Proc Cambr Philos Soc 1930, 26, 376.

5. Howard, I. A.; March, N. H.; Senet, P.; Van Doren, V. E. Phys Rev A: At Mol Opt Phys 2000, 62, 062512.

6. March, N. H. Int J Quantum Chem 2003, 92, 1.

7. Layzer, D. Ann Phys (NY) 1959, 8, 271.

8. Cohen, M. Adv At Mol Phys 1988, 25, 195.

9. March, N. H. Phys Rev A: At Mol Opt Phys 1986, 34, 5104.

10. See, for example, March, N. H. Self-Consistent Fields in Atoms; Pergamon: Oxford, 1975.

11. Kleinman, L. Phys Rev B 1994, 49, 14197.

12. Holas, A.; March, N. H. Phys Rev B 1997, 55, 1295.

13. Sharp, R. T.; Horton, G. K. Phys Rev 1955, 90, 316.

14. Talman, J. D.; Shadwick, W. F. Phys Rev A: At Mol Opt Phys 1976, 14, 36.

15. Harbola, M. K.; Sahni, V. Phys Rev Lett 1989, 62, 489.

16. Ou-Yang, H.; Levy, M. Phys Rev A: At Mol Opt Phys 1990, 41, 4038.

17. Holas, A.; March, N. H. Phys Rev A: At Mol Opt Phys 1995, 51, 2040.

18. March, N. H.; Howard, I. A.; Holas, A.; Senet, P.; Van Doren, V. E. Phys Rev A: At Mol Opt Phys 2001, 63, 012520.

19. Howard, I. A.; March, N. H.; Talman, J. D., accepted for publication, Phys Rev A 2003, 68, 044502.

20. Howard, I. A.; March, N. H. J Chem Phys 2003, 119, 5789.

21. Della Sala, F.; Görling, A. J Chem Phys 2001, 115, 5718.

22. Talman, J. D. Comp Phys Commun 1989, 54, 85.

23. Levy, M.; Perdew, J. P. Phys Rev A: At Mol Opt Phys 1985, 32, 2010.

24. Howard, I. A.; March, N. H.; Alonso, J. A.; Cordero, N. A.; Van Doren, V. E. Chem Phys Lett 2001, 343, 166.

25. Dawson, K. A.; March, N. H. J Chem Phys 1984, 81, 5.

26. March, N. H.; Santamaria, R. Int J Quantum Chem 1991, 39, 585.

27. Lee, H.; Lee, C.; Parr, R. G. Phys Rev A: At Mol Opt Phys 1991, 44, 768. 